\title{
Kinetische Untersuchungen zur Gamma-Radiolyse von 5-Bromuracil in wäßriger Lösung
}

\author{
H. REUSCHL \\ Institut für Strahlenbiologie, Kernforschungszentrum Karlsruhe \\ (Z. Naturforschg. 21 b, 643-646 [1966]; eingegangen am 10. März 1966)
}

\begin{abstract}
Es wurde die Kinetik des Abbaus von 5-Bromuracil in wäßriger Lösung bei $p_{\mathrm{H}}=6,5$ durch Bestrahlung mit $\gamma$-Strahlen unter $\mathrm{N}_{2}, \mathrm{O}_{2}$ und $\mathrm{N}_{2} \mathrm{O}$ untersucht. Die Ergebnisse werden an Hand der Radikaltheorie wäßriger Lösungen diskutiert und eine Hypothese zur Ursache der Strahlensensibilisierung lebender Zellen durch Bromuracil abgeleitet.
\end{abstract}

Nach dem Einbau von 5-Bromuracil (BU) anstelle von Thymin ( $\mathrm{T}$ ) in die DNS von lebenden Zellen oder von Phagen wird die Inaktivierungsrate derselben nach Bestrahlung mit ionisierenden Strahlen beträchtlich erhöht ${ }^{1}$. Diese „Sensibilisierung “ könnte unter anderem durch die unterschiedliche Strahlenempfindlichkeit von $\mathrm{BU}$ und $\mathrm{T}$ in der DNS bedingt sein, aber auch durch die Natur der Radiolyseprodukte, die nicht in jedem Fall eine letale Wirkung haben und in unterschiedlichem Ausmaß eine Reaktivierung der geschädigten DNS zulassen werden. So erhält die Frage nach dem Mechanismus der Sensibilisierung durch BU ihr Interesse vor allem durch die Möglichkeit, allein durch den Vergleich des unterschiedlichen strahlenbiologischen Verhaltens zweier in gewissem $\mathrm{Maß}$ einander ersetzender Basen Einblicke in grundlegende strahleninduzierte chemisch-physikalische Reaktionen in der Zelle zu gewinnen.

Für den Fall der Strahlensensibilisierung von BU-haltigen Enterococcus Stei („schweren“ Zellen) durch Röntgenstrahlen wurde bereits von LocHMANN $^{2}$ an Hand der Basen-Analyse von normalen und „schweren“ Zellen eine Erklärung dieser Erscheinung vorgeschlagen: Nach Verabreichung der gleichen Dosis $(\sim 1000 \mathrm{kr})$ war der prozentuale Abbau des BU in den schweren um das 5-10-fache höher als der des $\mathrm{T}$ in normalen Zellen; als Umwandlungsprodukt des BU wurde fast ausschließlich Uracil (U) gefunden. Die vorgeschlagene Erklärung für die Ursache der Sensibilisierung gründet sich auf die Annahme, die Bildung von U sei eine letale Mutation. Der Mechanismus, nach dem sich das U bildet, bleibt ungeklärt, ebenso wie es unbewiesen

1 B. Djordjevic u. W. Szybalski, J. exp. Medicine 112, 509 [1960]; G. Нотz u. K. G. Zimmer, Int. J. Rad. Biol. 7, 75 [1963]; F. W. Stahl et al., Virology 13, 98 [1961]. bleibt, ob U in der DNS dieser Zellart letal wirkt.

In der vorliegenden Arbeit soll versucht werden, durch die Untersuchung der Kinetik des Abbaus von BU bei Bestrahlung unter $\mathrm{N}_{2}, \mathrm{O}_{2}$ und $\mathrm{N}_{2} \mathrm{O}$ mit den $\gamma$-Strahlen von ${ }^{60} \mathrm{Co}$ einen ersten globalen Überblick über die auftretenden strahlenchemischen Reaktionen zu gewinnen.

\section{Experimenteller Teil}

BU wurde in Konzentrationen zwischen $1 \cdot 10^{-4}$ $3 \cdot 10^{-3} \mathrm{Mol} / l$ in dreimal dest. Wasser gelöst und mit einer $\mathrm{Na}_{2} \mathrm{HPO}_{4} / \mathrm{NaH}_{2} \mathrm{PO}_{4}$-Pufferlösung von $p_{\mathrm{H}}=6,5$ versetzt, so daß die Konzentration des Puffersalzes doppelt so hoch war wie die des BU. Zur Bestrahlung unter $\mathrm{N}_{2}$ wurden die BU-Lösungen in Ampullen 20 Min. mit $\mathrm{O}_{2}$-freiem $\mathrm{N}_{2}$ gespült und abgeschmolzen. Bei den Versuchen mit $\mathrm{O}_{2}$ leiteten wir während der Bestrahlung $\mathrm{O}_{2}$ durch die Lösungen, so daß die $\mathrm{O}_{2}$-Konzentration konstant und gleich der Sättigungskonzentration war: $1,3 \cdot 10^{-3} \mathrm{Mol} / l$. $\mathrm{N}_{2} \mathrm{O}$ wurde durch Ausfrieren bei $-80{ }^{\circ} \mathrm{C}$ und Abpumpen der nicht kondensierten Gase $\left(\mathrm{O}_{2}\right)$ gereinigt und in einer geschlossenen Glasapparatur über die vorher durch freeze-pumping entgasten BU-Lösungen geleitet. Nach Abschmelzen der die Lösungen enthaltenen Ampullen und Einstellen des Gasgleichgewichts betrug der Partialdampfdruck des $\mathrm{N}_{2} \mathrm{O}$ $350 \pm 30 \mathrm{~mm} \mathrm{Hg}$, dies entspricht einer Konzentration des $\mathrm{N}_{2} \mathrm{O}$ in der BU-Lösung von etwa $1 \cdot 10^{-2} \mathrm{Mol} / l^{3}$. Die Bestrahlung erfolgte in einer ${ }^{60} \mathrm{Co}$-Quelle mit einer Dosisleistung von $6 \cdot 10^{5} \mathrm{r} /$ Stunde.

Die Menge des nach Bestrahlung unzerstörten BU wurde durch Messung der Lichtabsorption bei $274 \mathrm{~m} \mu$ auf parallelem Wege direkt und nach erfolgter chromatographischer Isolierung des BU von den Radiolyseprodukten ermittelt. Es stellte sich heraus, daß bei Bestrahlung unter $\mathrm{O}_{2}$ die direkt und die nach Chromatographie gefundenen Werte innerhalb der Fehlergrenze des letzten Verfahrens übereinstimmten, so daß es in

2 E. R. Lochmans, Naturwissenschaften 50, 520 [1963].

3 K. D. Asmus u. A. Henglein, Ber. Bunsenges. physik. Chem. 68, 348 [1964]. 
diesem Falle möglich war, die genauere direkte Messung anzuwenden; die Fehlerbreite beträgt etwa \pm 3 Prozent. Wie der Vergleich der beiden Messungen zeigte, bilden sich dagegen bei Bestrahlung unter $\mathrm{N}_{2}$ und $\mathrm{N}_{2} \mathrm{O}$ UV-absorbierende Stoffe und man erhält erst nach chromatographischer Trennung gültige Werte für den Abbau des BU. Vor dem Auftragen auf das Chromatogramm mußten die Lösungen konzentriert werden; dies erfolgte durch Gefriertrocknung und Lösen der getrockneten Substanz in einem entsprechend kleineren Volumen Wassers. Zur chromatographischen Trennung wurden Kieselgelschichten verwendet, als Laufmittel diente ein Gemisch von Äthylacetat und 65-proz. wässrigem Isopropanol $75: 25$. Als Fehlerbreite für die Bestimmung des BU ergab sich etwa \pm 8 Prozent.

\section{Ergebnisse und Diskussion}

Die Abnahme der BU-Konzentration in Abhängigkeit von der Dosis bei Bestrahlung in wäßriger Lösung unter $\mathrm{N}_{2}, \mathrm{O}_{2}, \mathrm{~N}_{2} \mathrm{O}$ läßt sich bis zu einem Abbaugrad von etwa $50 \%$ in halblogarithmischer Auftragung als Gerade graphisch darstellen (Abbn. 1, 2, 3). Die bei der Bestrahlung unter $\mathrm{N}_{2}$ und $\mathrm{N}_{2} \mathrm{O}$ erhaltenen initialen $G$-Werte sind innerhalb der Fehlergrenzen der BU-Bestimmung unabhängig von der Ausgangskonzentration des BU (Abb.4) und betragen im Mittel

$$
G_{-\mathrm{Bu}}^{\left(\mathrm{N}_{2}\right)}=7,50 \quad \text { und } \quad G_{-\mathrm{Bu}}^{\left(\mathrm{N}_{2} \mathrm{O}\right)}=5,50 .
$$

Bei der Bestrahlung mit $\mathrm{O}_{2}$ steigt dagegen der $G$ Wert mit der Anfangskonzentration des BU stark

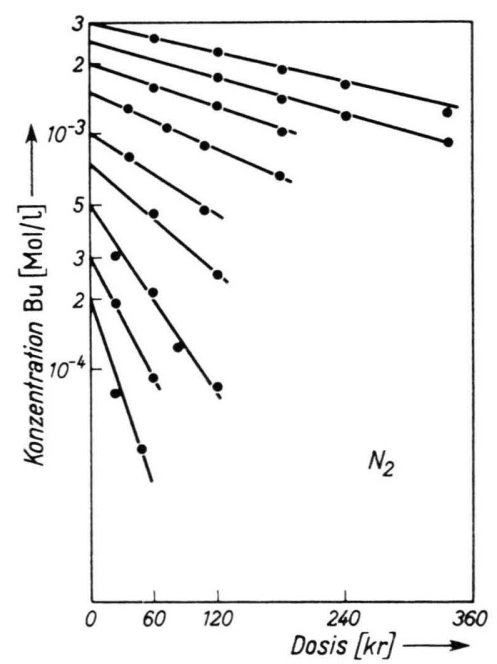

Abb. 1. Strahlenchemischer Abbau des BU in wäßriger Lösung bei verschiedenen Konzentrationen durch $\gamma$-Bestrahlung in Gegenwart von $\mathrm{N}_{2}$.

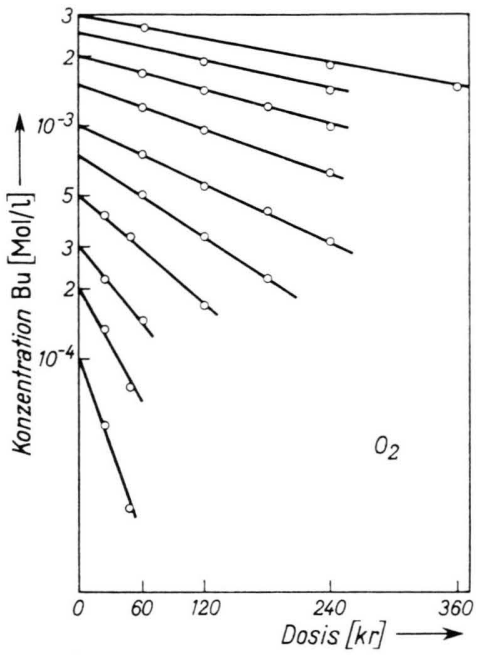

Abb. 2. Abbau des BU in wäßriger Lösung bei verschiedenen Konzentrationen durch $\gamma$-Bestrahlung in Gegenwart von $\mathrm{O}_{2}$.

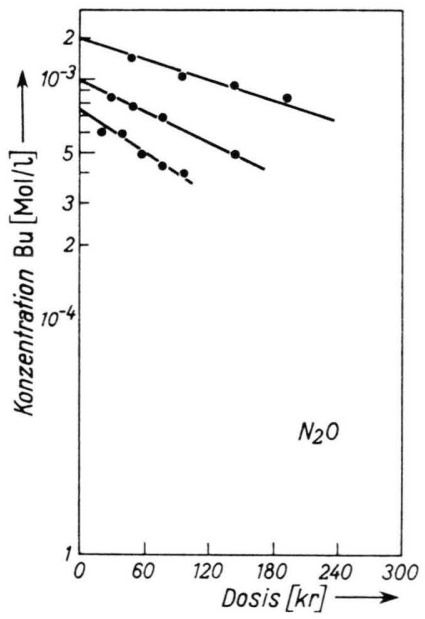

Abb. 3. Abbau des BU in wäßriger Lösung bei verschiedenen Konzentrationen durch $\gamma$-Bestrahlung in Gegenwart von $\mathrm{N}_{2} \mathrm{O}$.

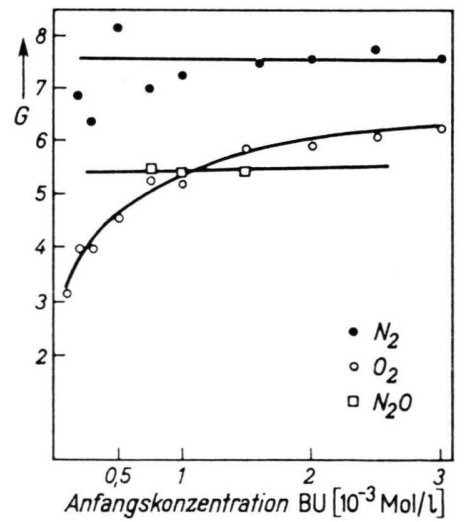

Abb. 4. Abhängigkeit der $G$-Werte des Abbaus des BU von der Konzentration bei $\gamma$-Bestrahlung wäßriger BU-Lösungen in Gegenwart von $\mathrm{N}_{2}, \mathrm{O}_{2}$ und $\mathrm{N}_{2} \mathrm{O}$. 
an (Abb. 4); die extremen gemessenen Werte betragen bei

$$
\begin{aligned}
& (\mathrm{BU})_{0}=1 \cdot 10^{-4} \mathrm{Mol} / l: G_{-\mathrm{Bu}}^{\left(\mathrm{O}_{2}\right)}=3 \quad \text { und bei } \\
& (\mathrm{BU})_{0}=3 \cdot 10^{-3} \mathrm{Mol} / l: G_{-\mathrm{Bu}}^{\left(\mathrm{O}_{2}\right)}=6,4 .
\end{aligned}
$$

Diese $G$-Werte lassen bei Berücksichtigung der zuletzt von $\mathrm{H}_{\text {Ayon }}{ }^{4}$ für die Radiolyse wäßriger Lösungen ermittelten $G$-Werte der "Wasserradikale“ eine sehr einfache Deutung zu. Danach reagieren in verdünnten wäßrigen Lösungen bei neutralem $p_{\mathrm{H}}$-Wert die bei der Radiolyse des Wassers gebildeten Species $\mathrm{H}^{*}, \mathrm{OH}^{*}, \mathrm{e}_{\mathrm{aq}}$ innerhalb eines definierten Konzentrationsbereichs entsprechend folgenden $G$-Werten mit dem gelösten Stoff (S)

$$
G_{\mathrm{H}}=0,55 ; \quad G_{\mathrm{OH}}=2,25 ; \quad G_{\mathrm{e}_{\mathrm{aq}}}=2,30 .
$$

Die Grenzen dieses Konzentrationsbereichs sind abhängig von der Geschwindigkeitskonstanten der Reaktion eines "Wasserradikals“ (R) mit dem gelösten Stoff $k_{(\mathrm{R}+\mathrm{S})}$ und durch die Beziehungen $k_{(\mathrm{R}+\mathrm{S})} C_{\mathrm{S}} \cong 10^{5}$ als untere und $k_{(\mathrm{R}+\mathrm{S})} C_{\mathrm{S}} \geqq 10^{7}$ als obere Grenze definiert. Während bei Konzentrationen $C_{\mathrm{S}}<10^{5} / k_{(\mathrm{R}+\mathrm{S})}$ die Radikalreaktionen mit dem gelösten Stoff zugunsten der Radikalrekombination abnehmen, erhöht sich ihr Ausmaß bei $C_{\mathrm{S}}>10^{7} /$ $k_{(\mathrm{R}+\mathrm{S})}$ auf Kosten der Rekombination der in der „spur" gebildeten Radikale. Bei einer Geschwindigkeitskonstante $k_{\mathrm{R}+\mathrm{S}}=1 \cdot 10^{10} \mathrm{Mol}^{-1} \mathrm{sec}^{-1}$ steigt dabei der $G$-Wert von $\mathrm{OH}^{\circ}$ und e $\mathrm{e}_{\mathrm{aq}}$ um etwa 0,3 bei einer Erhöhung der Konzentration um das 10-fache. Im Falle der Bestrahlung von wäßrigen $\mathrm{N}_{2} \mathrm{O}$-Lösungen reagiert das hydratisierte Elektron mit $\mathrm{N}_{2} \mathrm{O}$ unter Bildung von $\mathrm{OH}^{\bullet}$ Radikalen: $\mathrm{N}_{2} \mathrm{O}+\mathrm{H}_{2} \mathrm{O}^{\ominus} \rightarrow$ $\mathrm{N}_{2}+\mathrm{OH}^{\cdot}+\mathrm{OH}^{\ominus}$ mit einer Geschwindigkeitskonstanten von $8,7 \cdot 10^{9} \mathrm{Mol}^{-1} \mathrm{sec}^{-15}$. Bei der von uns angewandten $\mathrm{N}_{2} \mathrm{O}$-Konzentration $C_{\mathrm{N}_{2} \mathrm{O}}=1 \cdot 10^{-2} \mathrm{Mol} / l$ betragen daher die $G$-Werte von e $\mathrm{aq}_{\mathrm{aq}}$ und $\mathrm{OH}^{*}: \mathrm{e}_{\mathrm{aq}}=$ 2,$55 ; G_{\mathrm{OH}}=2,50$.

Für unsere Versuche unter $\mathrm{N}_{2}$ können wir in erster Annäherung annehmen, daß die untersuchten BU-Konzentrationen im Bereich der oben definierten verdünnten Lösungen liegen und daher die angegebenen $G$-Werte gelten. Würden die Wasserradikale, ohne miteinander zu interferieren, jeweils ein BU-Molekül abbauen, so wäre bei Bestrahlung unter $\mathrm{N}_{2}$ der $G$-Wert des BU-Abbaus gleich der Summe $G_{\mathrm{H}^{*}}+G_{\mathrm{OH}^{-}}+G_{\mathrm{e}_{\mathrm{aq}}}=5,10$. Wir finden $G_{-\mathrm{BU}}^{\left(\mathrm{N}_{2}\right)}$ $=7,50$. Man kommt nun diesem experimentellen $G$-Wert sehr nahe, wenn man in diese Summe die $G$-Werte von entweder $\mathrm{OH}^{*}$ oder $\mathrm{e}_{\mathrm{aq}}$ mit dem Fak- tor 2 einsetzt:

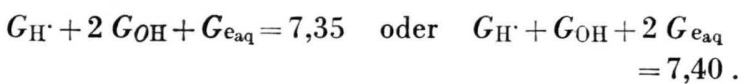

Folglich sollte entweder das $\mathrm{OH}^{-}$-Radikal oder das hydratisierte Elektron eine Zweistufenreaktion auslösen. Diese Frage konnte durch die Versuche mit $\mathrm{N}_{2} \mathrm{O}$ entschieden werden. Die Konzentration des $\mathrm{N}_{2} \mathrm{O}$ war im ungünstigsten Falle etwa sechsmal höher als die des BU und man kann daher bei der hohen Geschwindigkeitskonstanten der Reaktion $\mathrm{N}_{2} \mathrm{O}+e_{\mathrm{aq}}$ erwarten, daß sämtliche $\mathrm{e}_{\mathrm{aq}}$ von $\mathrm{N}_{2} \mathrm{O}$ abgefangen werden, bevor sie mit dem $\mathrm{BU}$ reagieren. Bei der angewandten Konzentration und unter der Annahme, daß je ein $\mathrm{OH}^{-}$-Radikal mit einem $\mathrm{BU}$ reagiert, wäre für den Abbau des BU ein $G$-Wert $G_{-\mathrm{BU}}^{\left(\mathbf{N}_{2} \mathrm{O}\right)}=0,55+2,50+2,55=5,6$ zu erwarten. Gefunden wurde $G_{-\mathrm{BU}}^{\left(\mathrm{N}_{2} \mathrm{O}\right)}=5,4$. Es ist somit gezeigt, daß bei der Radiolyse wäßriger BU-Lösungen die Radikale $\mathrm{H}^{*}$ und $\mathrm{OH}^{*}$ je ein, das hydratisierte Elektron jedoch in einer Folgereaktion zwei BU-Moleküle chemisch umwandeln.

Bei den Versuchen mit $\mathrm{O}_{2}$ bleibt es wegen der relativ niedrigen $\mathrm{O}_{2}$-Konzentration unsicher, in welchem Ausmaß die Reaktionen $\mathrm{e}_{\mathrm{aq}}+\mathrm{O}_{2} \rightarrow \mathrm{O}_{2}{ }^{\ominus}$ $\mathrm{H}^{*}+\mathrm{O}_{2}{ }^{\ominus} \rightarrow \mathrm{HO}_{2}{ }^{\cdot} \rightleftarrows \mathrm{O}_{2}{ }^{\ominus}+\mathrm{H}^{\oplus}$ mit den entsprechenden Reaktionen des BU konkurrieren. Die Tatsache, $\mathrm{da} ß$ bei einer Konzentration des $\mathrm{BU}=3 \cdot 10^{-3}$ der $G$-Wert des BU-Abbaus mit 6,4 höher liegt als der der einfachen Summe der $G$-Werte der Wasserradikale zeigt jedoch, daß bei dieser Konzentration in gewissem Umfang noch eine Zweistufenreaktion stattfindet. Da bei kleiner BU-Konzentration das Ausmaß des Abbaus sich stark verringert, wird diese Zweistufenreaktion nicht durch das $\mathrm{O}_{2}{ }^{\ominus}$ ausgelöst, sondern durch die von der Konkurrenzreaktion des $\mathrm{O}_{2}$ nicht abgefangenen $\mathrm{e}_{\mathrm{aq}}$. Dieser Befund spricht für eine große Reaktivität des $\mathrm{e}_{\mathrm{aq}}$ mit dem $\mathrm{BU}$, da die Geschwindigkeitskonstante der Reaktion $\mathrm{O}_{2}+\mathrm{e}_{\mathrm{aq}}$ mit $k_{0_{2}}+\mathrm{e}_{\mathrm{aq}}=2 \cdot 10^{-10} \mathrm{Mol}^{-1} \mathrm{sec}^{-1} \mathrm{sehr}$ hoch liegt. Die Frage, ob das $\mathrm{BU}$ von $\mathrm{O}_{2}{ }^{\ominus}$ angegriffen wird, kann durch diese Versuche nicht beantwortet werden.

Aus unseren Untersuchungen ist bei Berücksichtigung des strahlenchemischen Verhaltens von Pyrimidin-Verbindungen einerseits und Halogenverbindungen andererseits ein allgemeines Schema für den Reaktionsablauf bei der Radiolyse wäßriger BU.

\footnotetext{
${ }^{4}$ E. Hayon, Trans. Faraday Soc. 61, 723 [1965].

5 F. S. Dainton u. D. C. W $\mathrm{W}_{\mathrm{Alk}}$, Proc. Roy. Soc. [London], Ser. A 285, 339 [1964].
} 
Lösungen in Anwesenheit von Radikalfängern zwanglos abzuleiten. Ausgangspunkt ist dabei der Befund, daß die Radikale $\mathrm{H}^{\circ}$ und $\mathrm{OH}^{\circ}$ mit dem $\mathrm{BU}$ ein relativ stabiles Radikal bilden, das sich durch Reaktionen mit anderen Radikalen zu einem neutralen Molekül umwandelt, während sich mit dem hydratisierten Elektron ein sehr reaktives Radikal bildet, das ein weiteres BU-Molekül angreift. Von UracilDerivaten (z. B. Thymin) ist bekannt, daß sie bei Bestrahlung in wäßriger Lösung vorzugsweise $\mathrm{H}^{*}$ und $\mathrm{OH}^{-}$an die 5.6-Doppelbindungen addieren ${ }^{6}$; das gebildete Hydrouracilradikal<smiles>NC=CCC(C=O)C=O</smiles>

ist relativ stabil. Halogen-Derivate reagieren im allgemein sehr schnell mit dem hydratisierten Elektron unter Abspaltung des Halogens als Anion und Bildung eines Radikals. Im Falle von Bromuracil würde sich also das Uracil-Radikal bilden

6 G. Scholes, Progr. Biophysics molecular Biol. 13, 60 [1963].

7 A. Kamal u. W. M. Garrison, Nature [London] 206, 1315 [1965].<smiles>NC1C=CC(C=O)CC1</smiles>

von dem eine hohe Reaktivität zu erwarten ist. Vergleicht man, im Hinblick auf die Strahlensensibilisierung BU-haltiger Zellen, das strahlenchemische Verhalten in wäßriger Lösung von $\mathrm{T}^{6}$ und $\mathrm{BU}$, so ist wohl der wichtigste Unterschied darin zu suchen, daß nur im Falle von BU ein sehr reaktives Radikal entsteht, das Uracilradikal, das fähig ist, ein weiteres neutrales Molekül abzubauen. Thymin addiert an die 5.6-Doppelbindung nicht nur $\mathrm{H}^{*}$ - und $\mathrm{OH}^{*}$ Radikale, sondern auch das hydratisierte Elektron ${ }^{7}$; es wurde aber bisher keine Reaktion zwischen einem Thyminradikal und einem neutralen Molekül nachgewiesen. Daher scheint die Hypothese berechtigt, als eine der Ursachen der Strahlensensibilisierung durch BU die Bildung des Uracilradikals in der Zelle anzusehen: Dieses Radikal könnte durch Reaktion mit neutralen Molekülen oder durch Zerfall diffusible Radikale erzeugen, die die Zelle über das in normalen Zellen festgestellte Ausmaß hinaus schädigen. 